\title{
اسهام الذذع السينمائية ( تقنية تزييف الوجوه) في البناء الجمالي في القلم السينمائي
}

\author{
عقيل الموسوي \\ السينما ـ الأكاديمية الدولية للفنون - بيلاروسيا \\ البريد الاكتروني: akeelakeel269@yahoo.com
}

يعد استخدام التقنيات الرقية في صلناعة السينماو توظيف الذكاء الصناعي من العوامل المهمة التي سـاعدة المخرجون السينمائيون في تطوير قدر اتهم الاخر اجية في صناعة الأفلام و تعد تقنية تزيف الوجوه او از الت الثيخوخة من التقنيات المهمة في معالجـة قصص السينما التي تتضمن شخصيات بفئات عمريـة مختلفة فيمكن تصوير ممثل بالتقنيات الرقمية ليظهر اصغر سنا بينما هو في الواقع بيدو كبير في السن ويمكن ايضا إعادة ممثل الى الحياة رغم كونه متوفي منذ زمن بعيد . . ومن هنا تناول البحث في فصله الأول مشكلة البحث وهي (كيف يمكن توظيف تقنيات تزيف الوجوه جماليا في مشاهد الفلم السينمائي)، اما أهمية البحث فكانت في افادة الطلبة والعاملين المتخصصين في المجال السينمائي من هذا البحث ، اما اهداف البحث فتلخصت في نقطتين هما : 1- الكثف عن اسهام الخدع السينمائية (تقنية تزييف الوجوه) جماليا في الفلم السينمائي. 2- الكثف عن التقنيات المستخدمة في صناعة الخدع السينمائية (تقنية تزييف الوجوه). اما الفصل الثاني الاطلار المنهجي : تضمن تعريف تقنية تزييف الوجوه و كيفية استخدامها في قصص الأفلام و الإمكانات التي يقدمها الممثل الافتر اضي المعالج بتقنية تزييف الوجوه في الفلم السينمائي. اما الفصل الثالث: تضمن وصفا لإجراءات البحث و التي اتبعها الباحث، كما تضمن البحث اختيـار عينة البحث و هي فلم (ترمنايتور : دارك فايت Terminator: Dark Fate-2019) وقام الباحث بتحليل عينة البحث معتمدا على النتائج التي خرج بها من الاطار النظري. و تضمن الفصل الر ابع: النتائج و الاستتناجات وخرج الباحث بتوصيات ومقترحسات و قائمـة للمصسادر في نهايـة

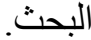

الكلمات المفتاحية: الخدع السينمائية، تزييف الوجوه، الفلم السينمائي. 


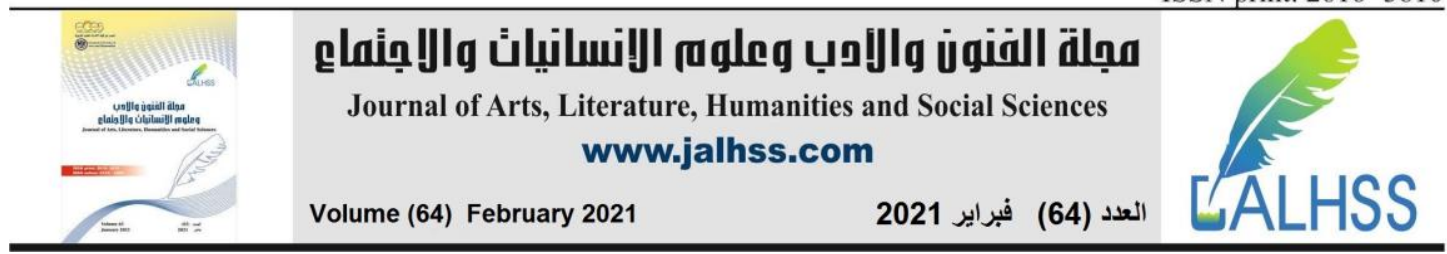

\title{
The Contribution of Cinematic Tricks
} (Technique of Falsifying Faces) to the Aesthetic Construction of the Cinematic Film

\author{
Akeel AL Mousawi \\ Cinema- International Academy of Arts - Belarus \\ Email: akeelakeel269@yahoo.com
}

\begin{abstract}
The use of digital technologies in the film industry and the employment of artificial intelligence is one of the important factors that helped filmmakers to develop their directing capabilities in filmmaking. Face falsification or aging is one of the important techniques in treating cinema stories that include characters of different age groups. Digital to show younger people when in reality they look old or bring an actor back to life despite being dead long ago.

Hence, the research in its first chapter dealt with the research problem, which is (How can techniques for aesthetically falsifying faces be employed in movie scenes). As for the importance of the research, it was in the benefit of students and workers specializing in the film field from this research. As for the objectives of the research, they were summarized in two points:

1-Exposing the aesthetic contribution of cinematic tricks (the technique of falsifying faces) in the cinematic film.

2 -Disclosure of the techniques used in making cinematic tricks (face counterfeiting technique).

As for the second chapter, the methodological framework: it included the definition of the face counterfeiting technique, how to use it in film stories, and the capabilities provided by the virtual actor who is treated with the facial counterfeiting technique in the cinematic film.

As for the third chapter: it included a description of the research procedures that the researcher followed, and the research also included choosing the research sample, which is the film (Terminator: Dark Fate 2019), and the researcher analyzed the research sample based on the results that he produced from the theoretical framework. The fourth chapter included: results and conclusions. The researcher came up with recommendations, proposals and a list of sources at the end of the research.

Cinematic Tricks, Falsifying Faces, The Motion Picture.
\end{abstract}

Keywords: Cinematic Tricks, Falsifying Faces, The Motion Picture. 


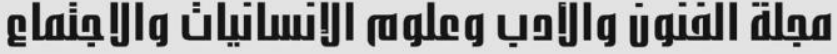

Journal of Arts, Literature, Humanities and Social Sciences www.jalhss.com

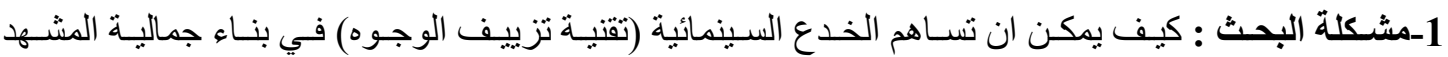

أـ الكشف عن التقنيات المستخدمة في صناعة الخدع السينمائية (تقنية تزييف الوجوه) بـ الكثف عن اسهام الخدع السينمائية ( تقنية تزييف الوجوه) في البناء الجمالي في الفلم السينمائي .

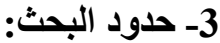

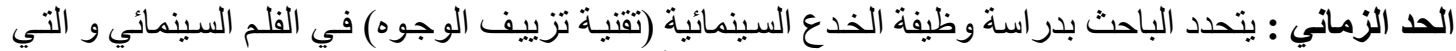

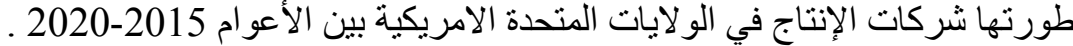

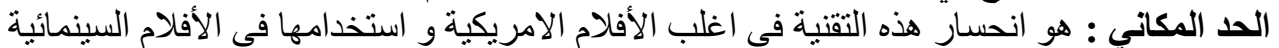

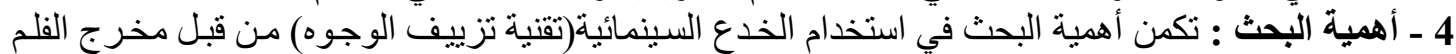

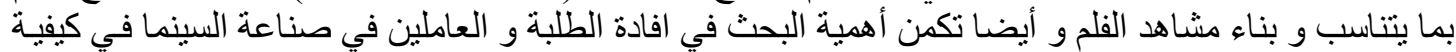

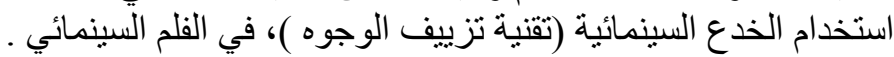

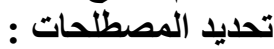

الخدعة السينمائية: ما يو هم به المشاهد بظنه حقيقة وهوة مجرد تقنتية سينمائية او طريقة مستخدمة في السينما

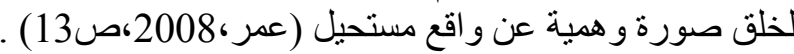

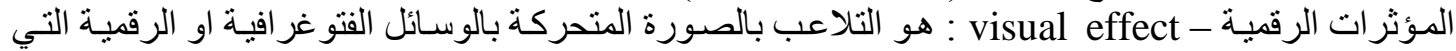
تخلق و هما و اقعيا لا يظهر في العالم الحقيقي (Mohammed,2012,p2 ).

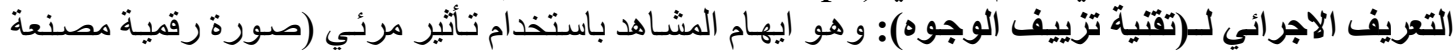

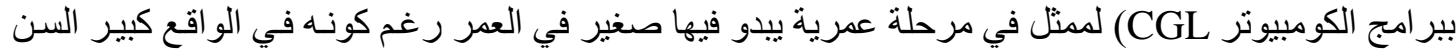
او متوفي و دمج هذه الصورة في مشهد سينمائي متحرك.

الفصل الثاني: الاطار النظري

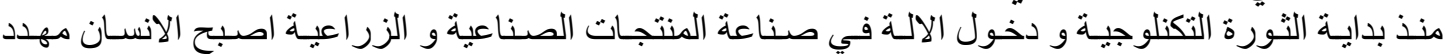

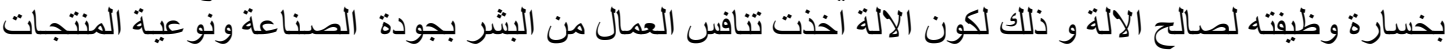

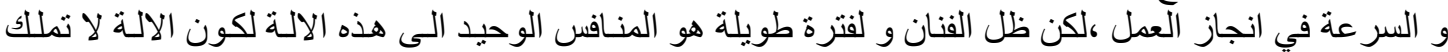

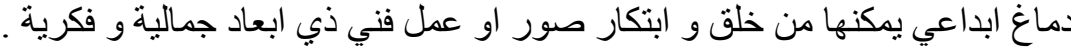

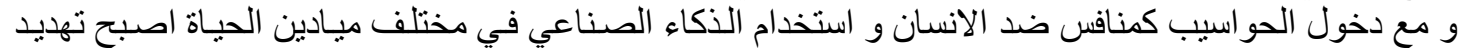

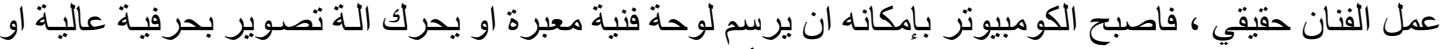

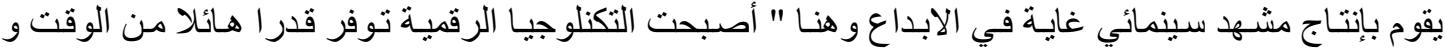

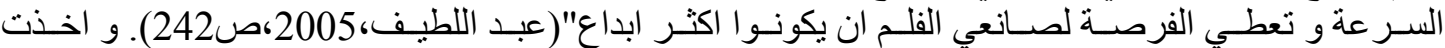

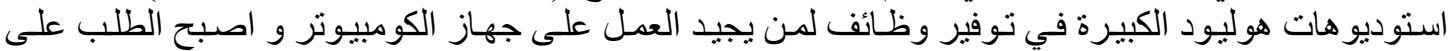

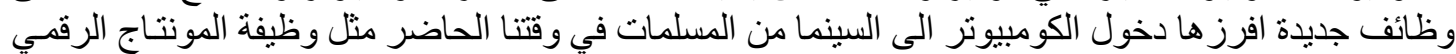

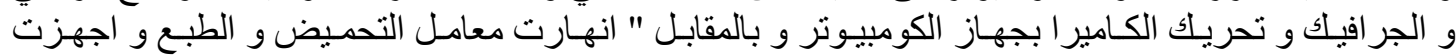

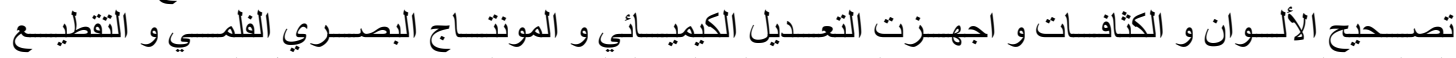

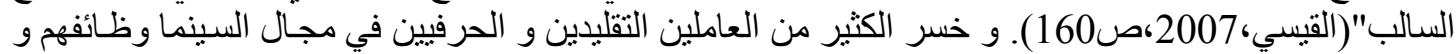

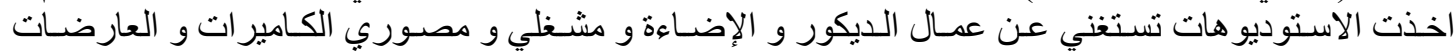

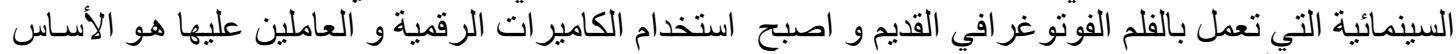

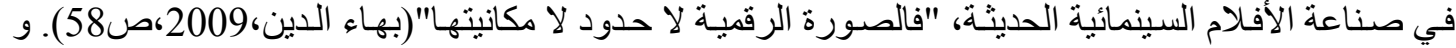

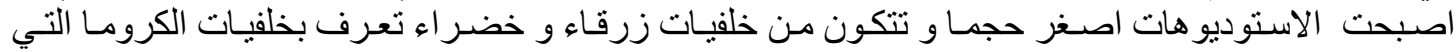

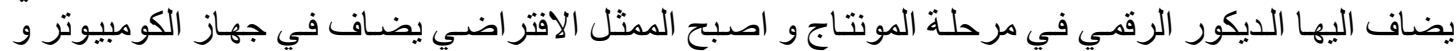

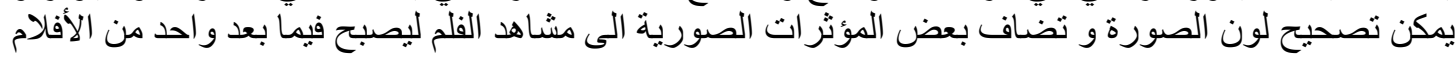




\section{هوالة القَو}

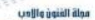

Journal of Arts, Literature, Humanities and Social Sciences

www.jalhss.com

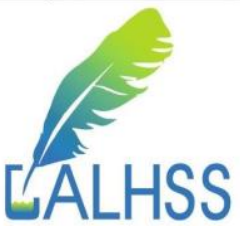

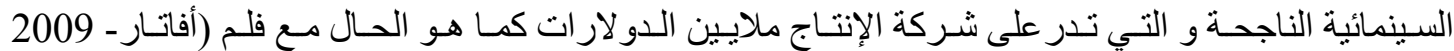

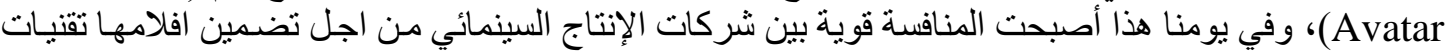

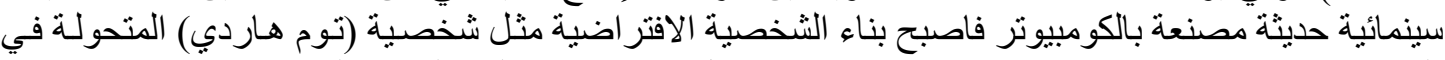

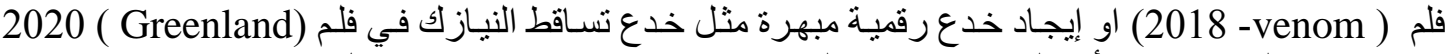

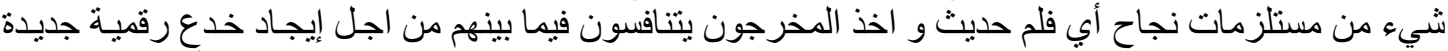

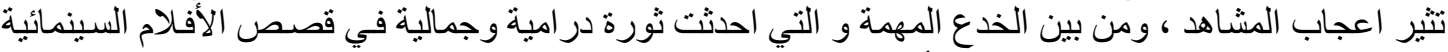

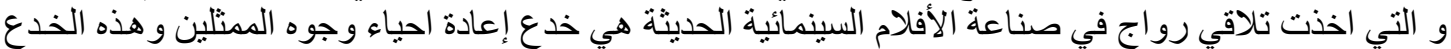

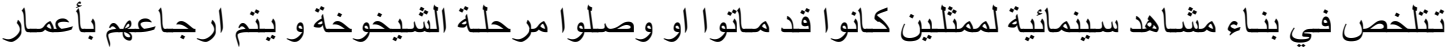

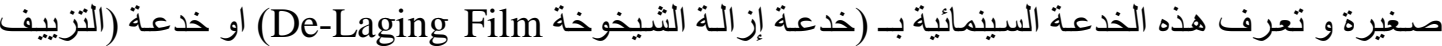
العميق Deep fake ) و هذه التسميتان هي لبر امج خدع سينمائية لها نفس النتائج و هو التحكم بملامح وجوه

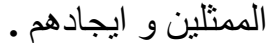
و أو ائل الثركات التي استخدمت هذه الخدع في افلامها هي شركة (لوكاس فلم ) فقامت بإنتاج صور رقميـة فنية

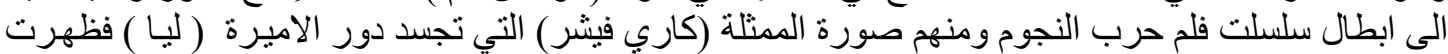

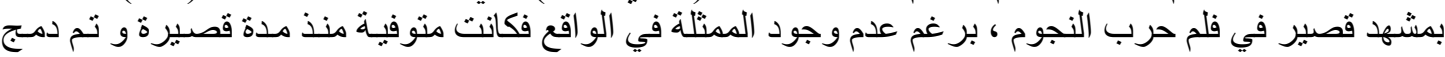

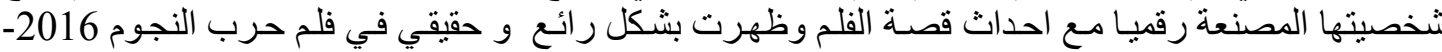
(Rogue One: A Star Wars Story)

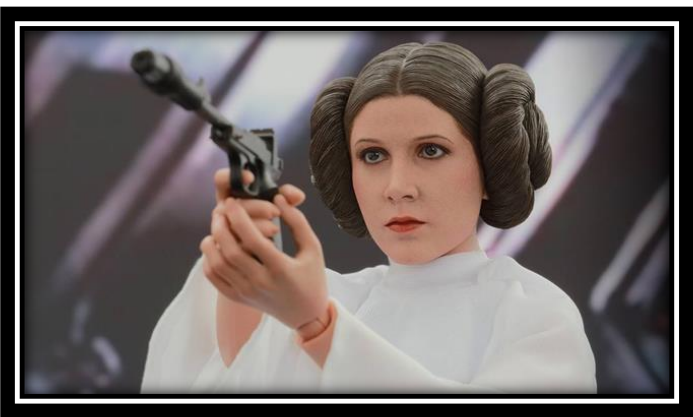

شكل رقم (1) (1) (1) (1)

$$
\begin{aligned}
& \text { الممثلة (كاري فيشر ) صورة معدلة } \\
& \text { ببر امج الكومبيوتر في فلم حرب }
\end{aligned}
$$

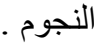

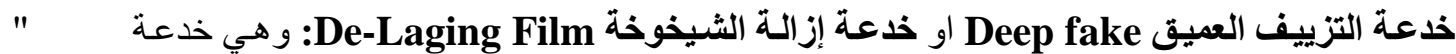

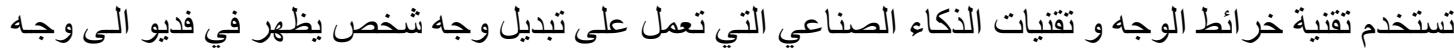

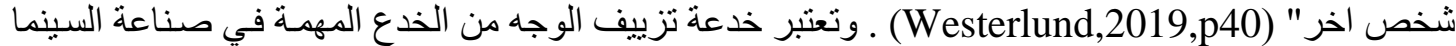

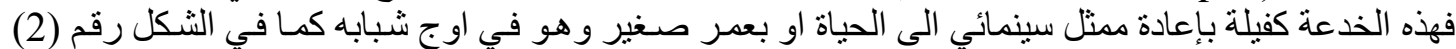
حيث يظهر الممثل صامويل جاكسون بعمر صغير في فلم كابتن مارفل .

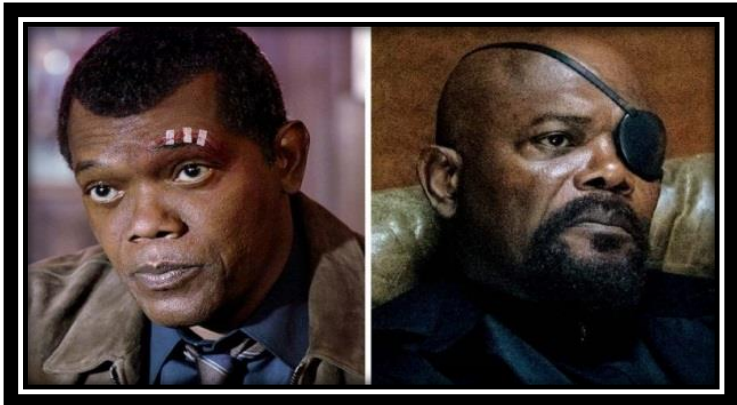

شكل رقم (2) بظهر صامويل جاكسون و هو صغير السن في فلم كابتن مارفل اما في الثكل

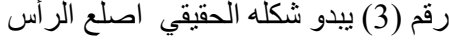
و لديه الكثير من التجاعيد وكبير في السن .

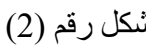

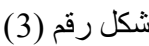




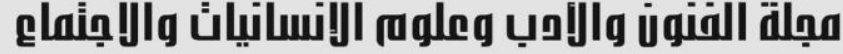

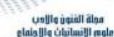

Journal of Arts, Literature, Humanities and Social Sciences www.jalhss.com

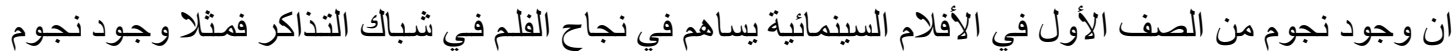

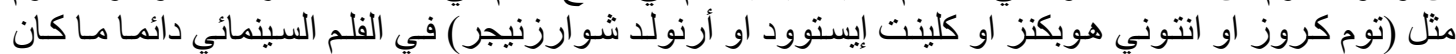

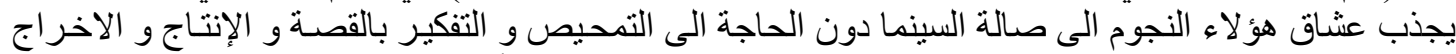

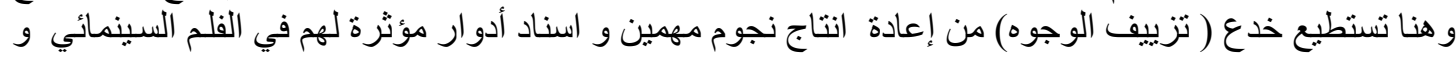

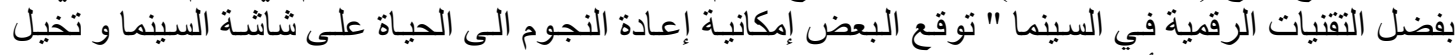

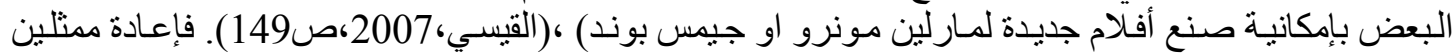

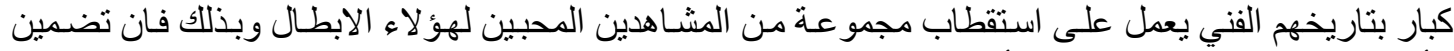

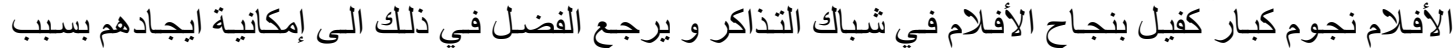

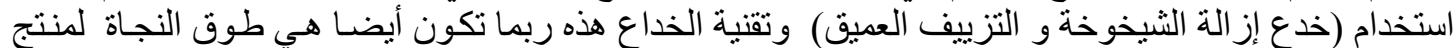

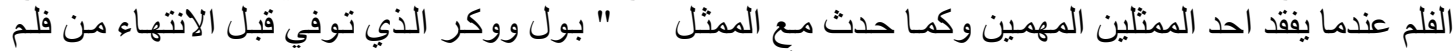

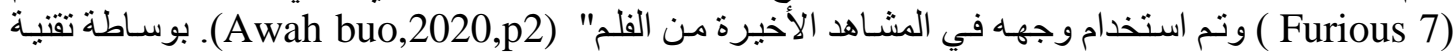

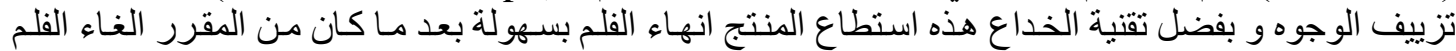

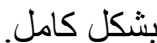

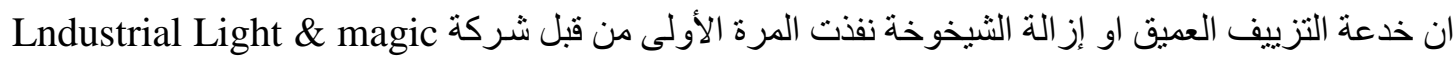

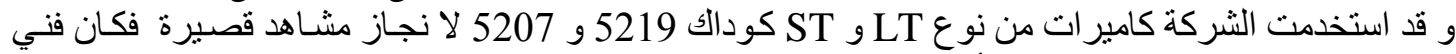

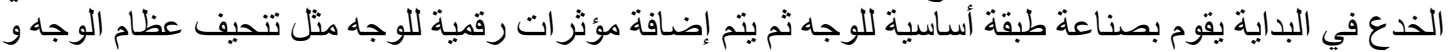

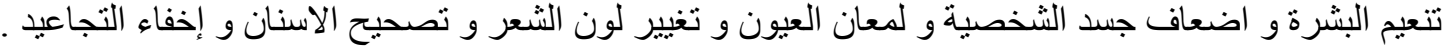

\section{طريقة عمل خدعة (تزييف الوجوه) :}

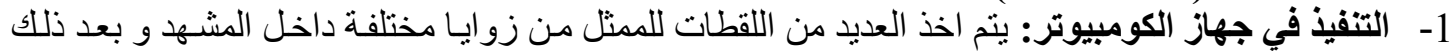

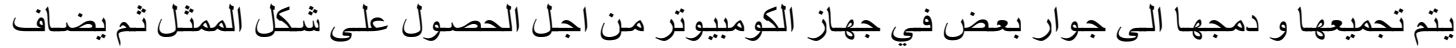

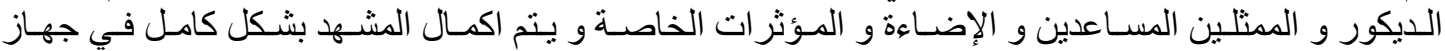

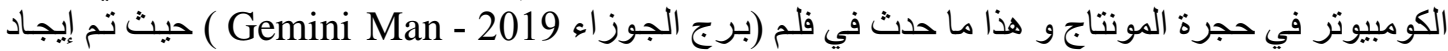

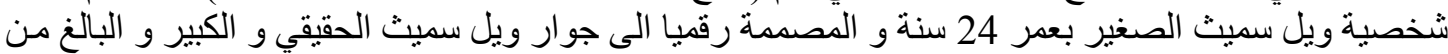

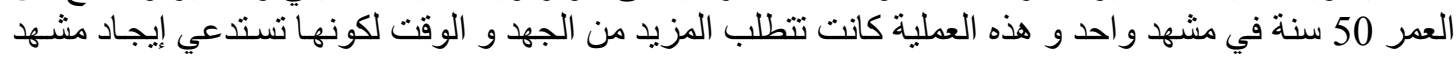

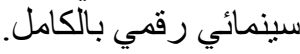
ويقول مشرف المؤثرات الخاصة غي وليامز " كان علينا البحث عن كل قطعة من اللقطات التي يمكن ان نجدها،

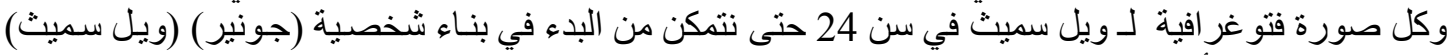

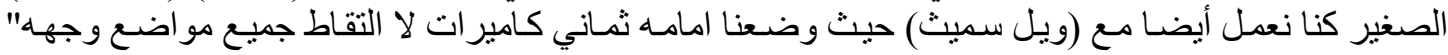

.(Burns, 2019)

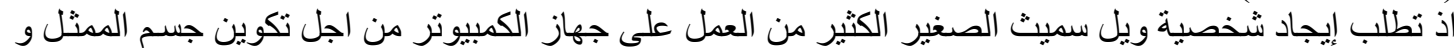

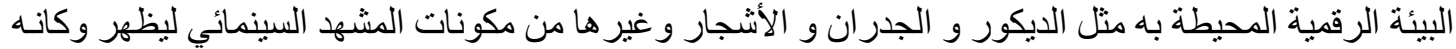

حقيقي بشكل كامل وكما يظهر في الثكل رقم (2).

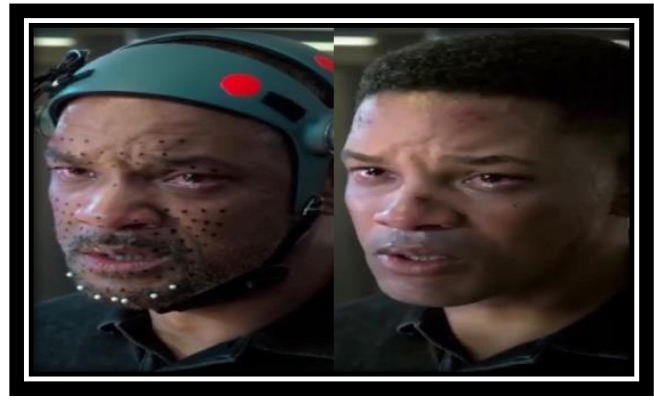

شكل رقم (4) الممثل (ويل سميث) الكبير

يؤدي دور (جونير) الصغير.

شكل رقم (4). 


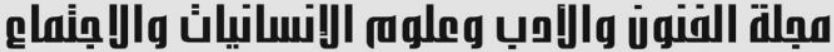

Journal of Arts, Literature, Humanities and Social Sciences

www.jalhss.com

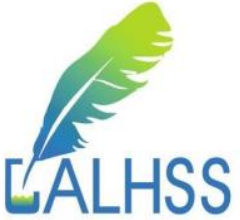

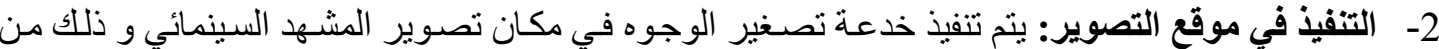

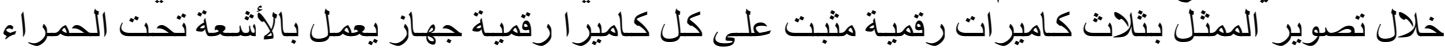

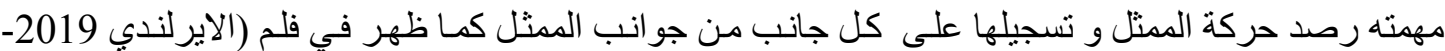

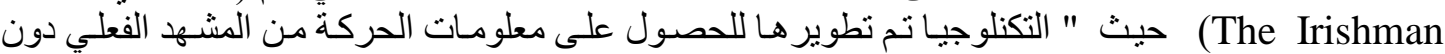

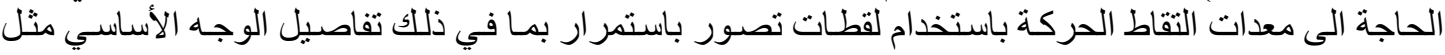

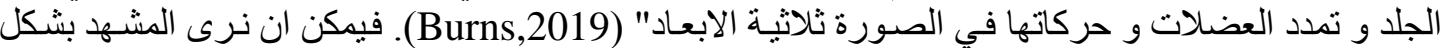

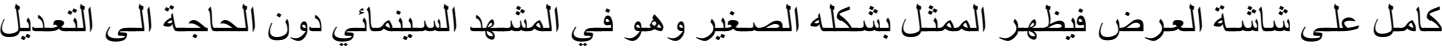

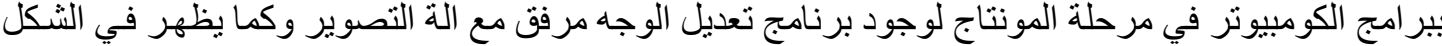

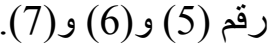

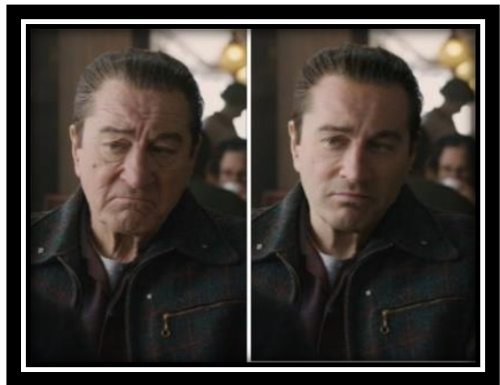

شكل رقم (7) المثنل روبرت دنيرو يبدو صغير السئن بعد تجميل وجهه بالخدع السينمائية

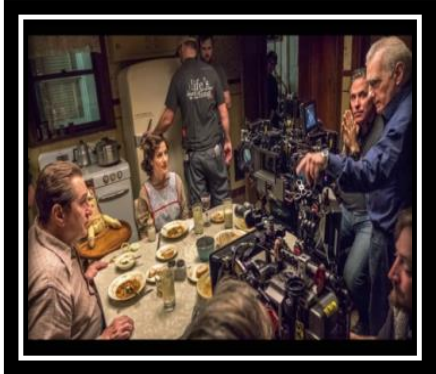

شكل رقم (6) كادر العمل

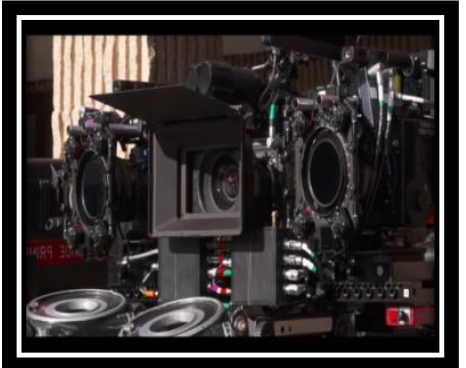

$$
\text { شكل رقم (5) الكامير ات }
$$

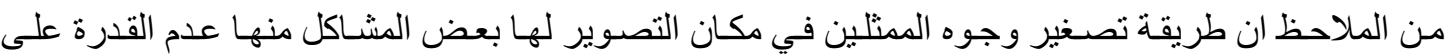

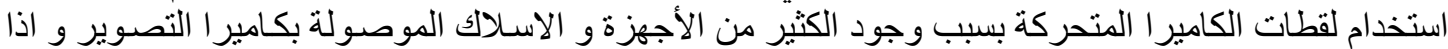

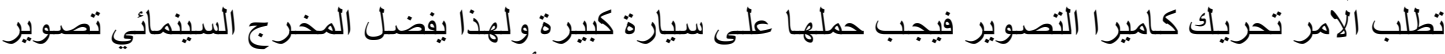

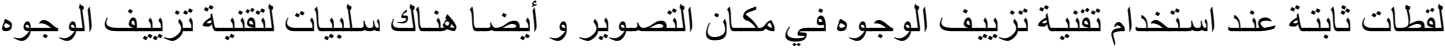

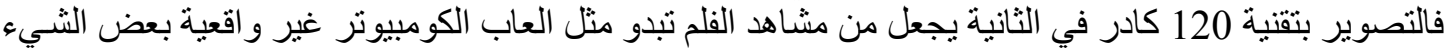

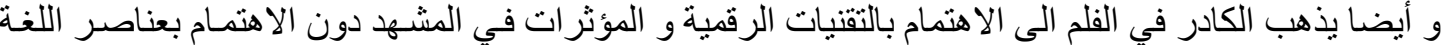
السينمائية مثل القصة و التمثيل و الإضلاءة و الإنيام الديكور.

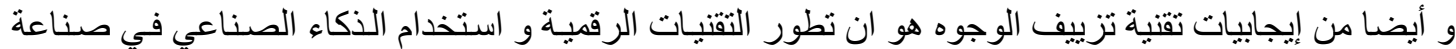

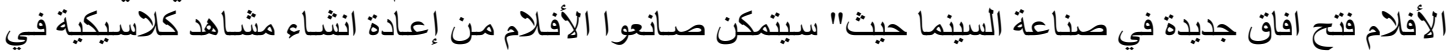

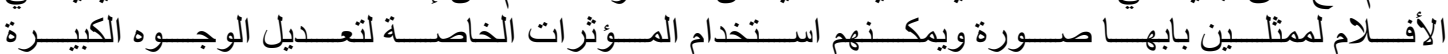

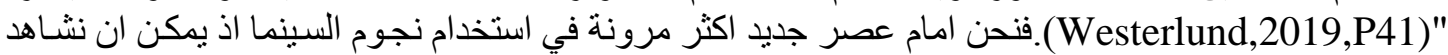

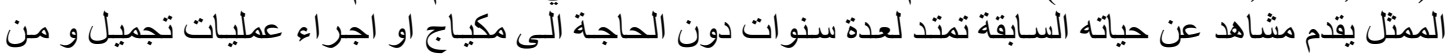

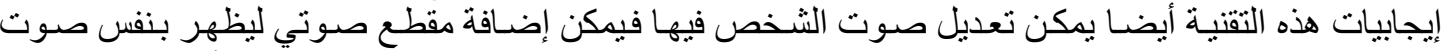

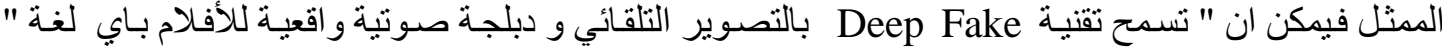
(Westerlund,2019,P41). فيمكن ان نشـاهد ممثل امريكي الجنسية يتحدث اللغـة العربية و العكس أيضـاو

$$
\text { بنفس مستوى طبقة الصوت ونبرة الصوت. }
$$

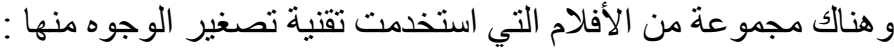

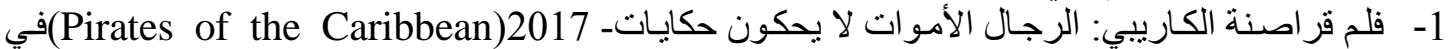

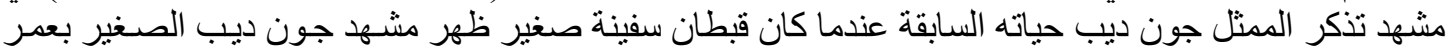

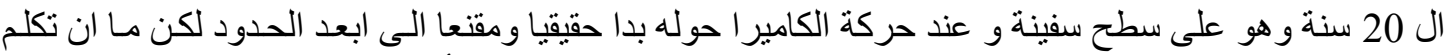

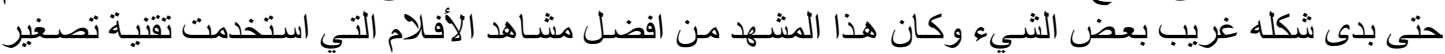




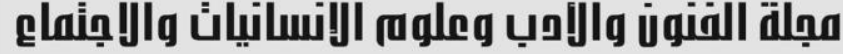

Journal of Arts, Literature, Humanities and Social Sciences www.jalhss.com

2- فلم (الرجل النملة Ant-Man -2015) ظهر الممثل مايكل دوجلاس صغير السن يختلف كثير ا عن شكله

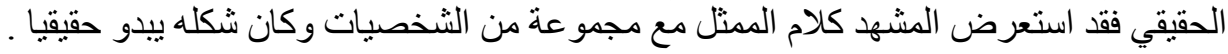

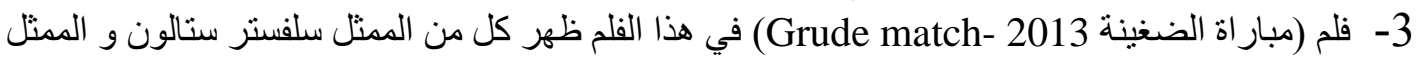

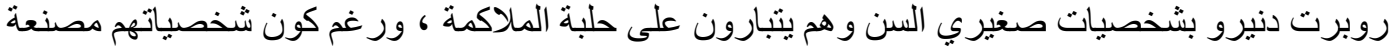

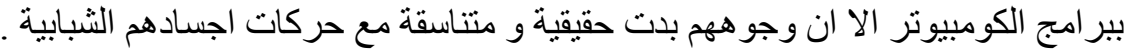

في النهاية ان استخدام تقنية (تزييف الوجوه قد يفرض بعض القو انين في صناعة السينما فقد تم التوقيع

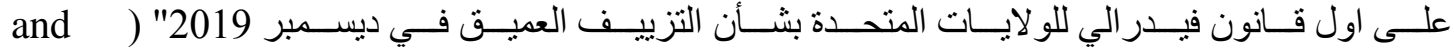
(Davis Wigging,2020,p3

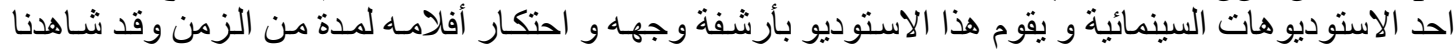

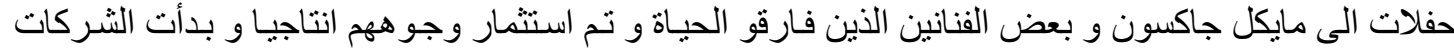

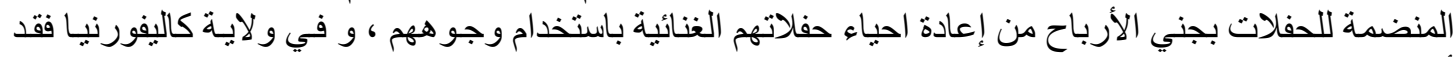

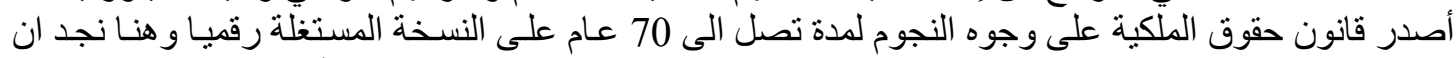

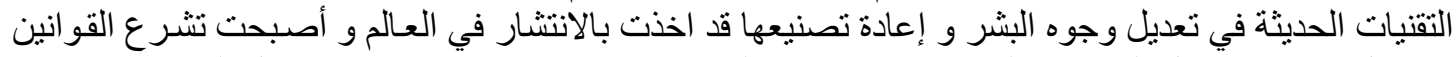

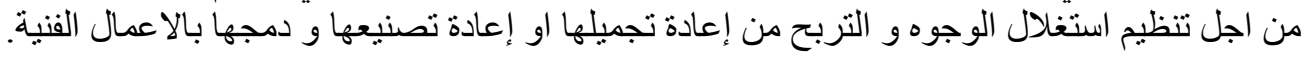

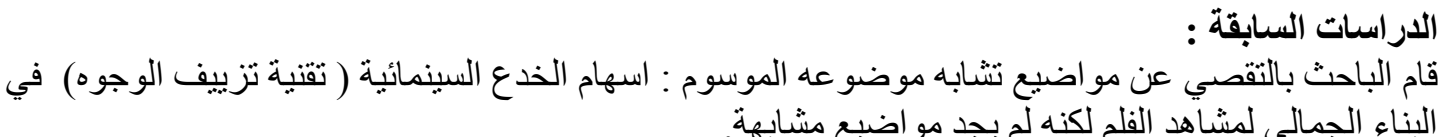

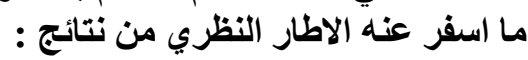

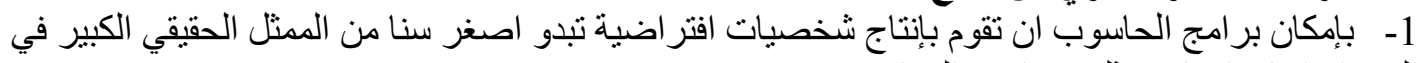
السن او إعادة احياء ممثلون غادر الادرو الحياة.

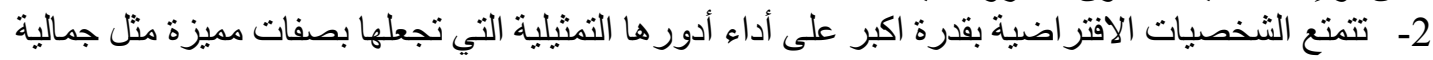

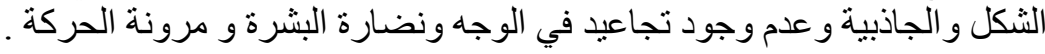

\section{الفصل الثالث إجراعات البحث}

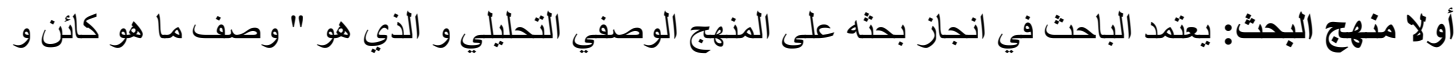

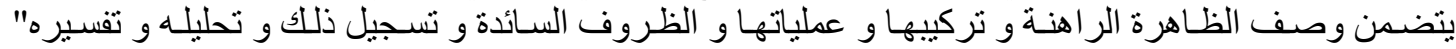

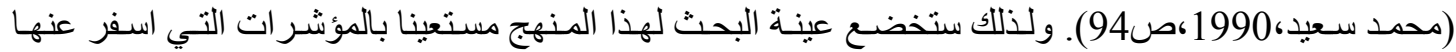

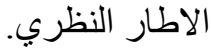

ثثانيا مجتمـع البحث: الأفلام الامريكية التي تحتوي في مضمونها شخصيات عوملت بتقنية الخدع السينمائية

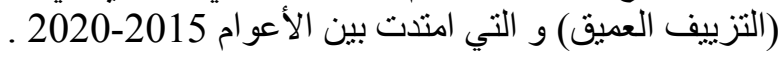

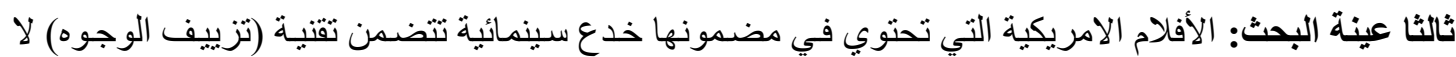

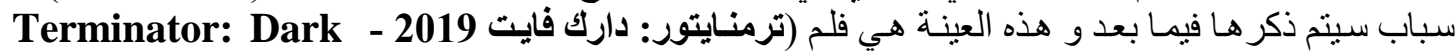
(Fate

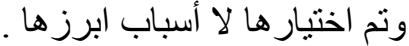
1- احتو اء هذا الفلم على الخدع السينمائية (تقنية تزييف الوجوه) التي تستخدم أنظمة رقمية حديثة .

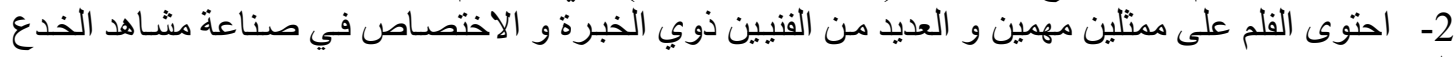

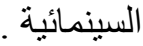




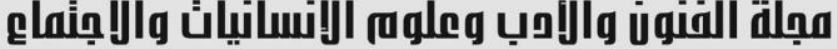

Journal of Arts, Literature, Humanities and Social Sciences www.jalhss.com

رابعا أداة البحث : لغرض تحقيق اعلى قدر من الموضعية و العلمية لهذه الدراسة فان البحث يتطلب وضع أداة و

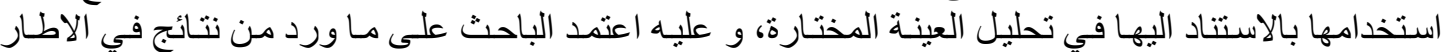
النظري كمؤشر ،لاستخدامهاً أداة التحليل .

خامسا - وحدة التحليل : اعتمد الباحث وحدة التحليل و هي المشهدو الذي يتفق و المؤشر الفني.

الفصل الرابع : تحليل العينة

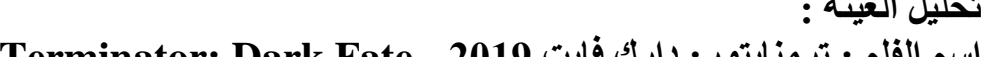

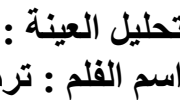

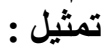

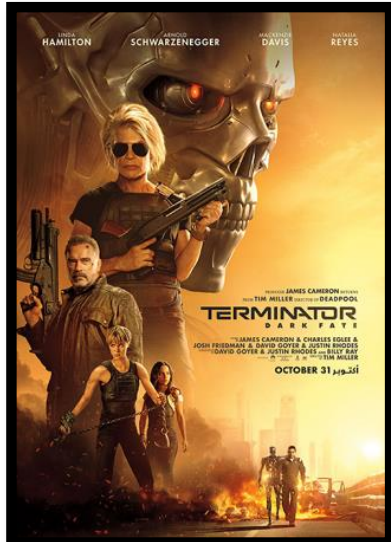

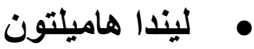

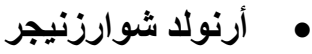
ماكنزي ديفيس ناتاليا رييس دائن جابرييل لوناليا ديجو بونيتا إخراج : تئم ميلر

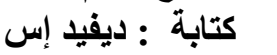

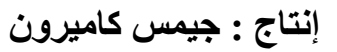

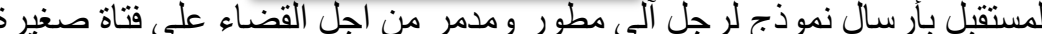

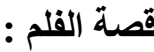

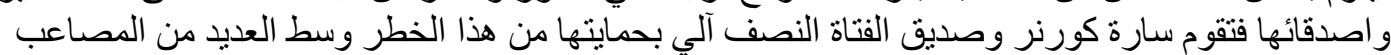

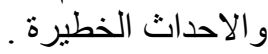

1- المؤشر الأول بإمكان الحاسوب ان يقوم بأنشاء شخصيات افتراضية معالجة بتقتيات تزييف الوجوه لتقوم

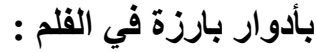

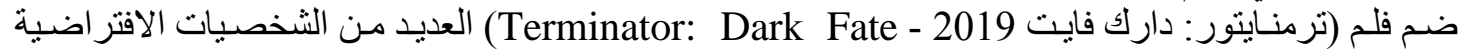

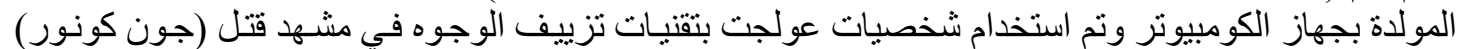

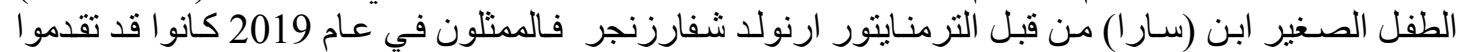

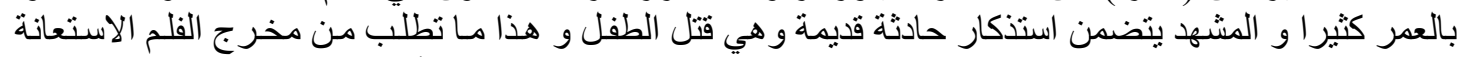

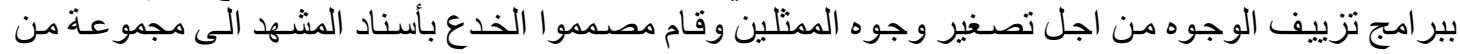

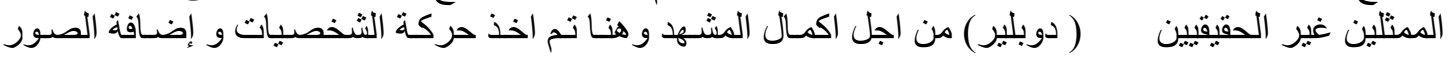

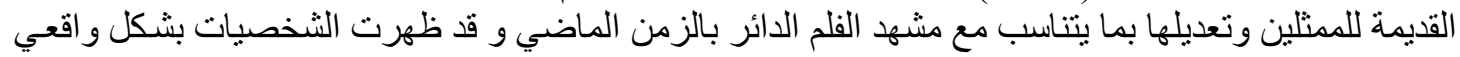
و مطابقة لفكرة الفلم وكما في الثكال (8)(9)(10).

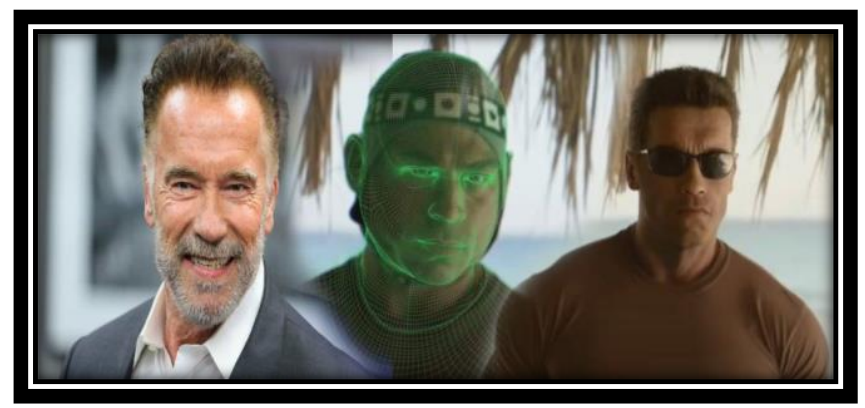

شكل رقم (8) تظهر الصورة الممثل ارنولا بشكله الحالي

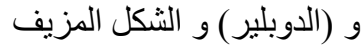
صغير السن . 


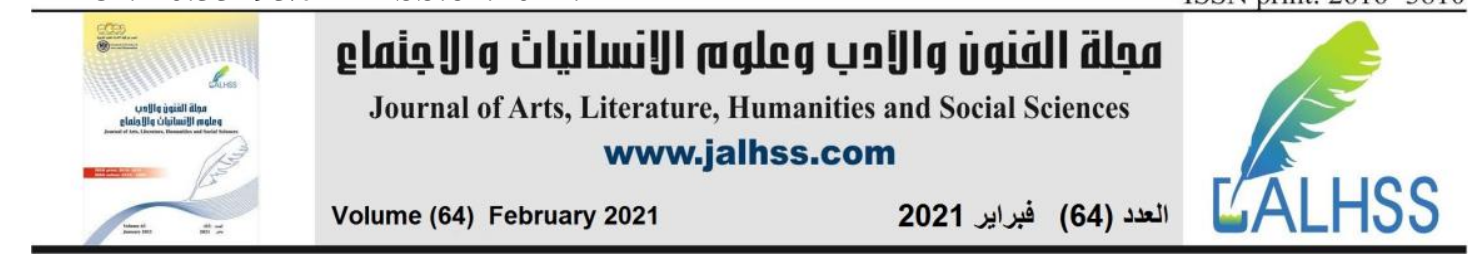

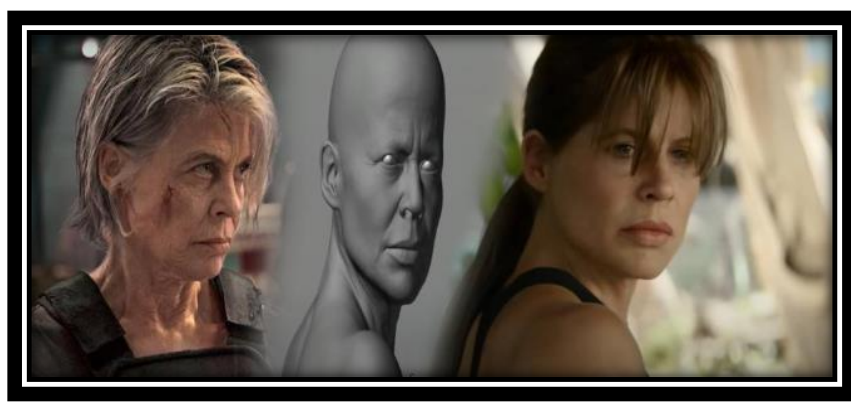

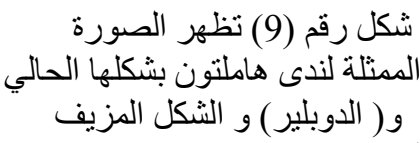

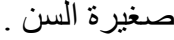

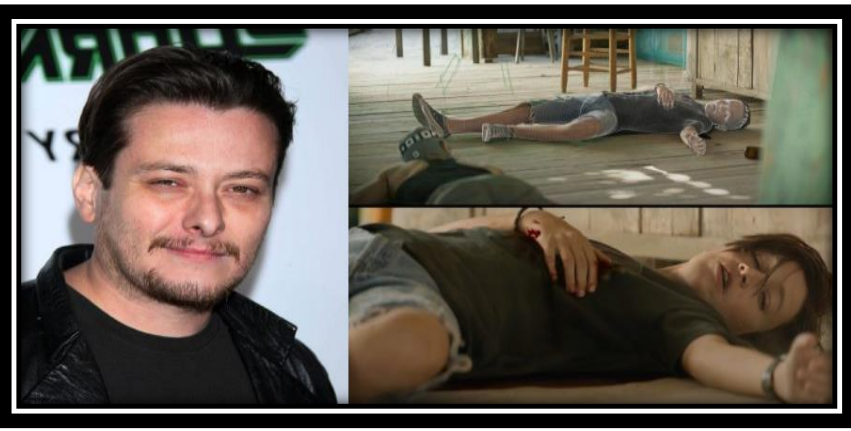

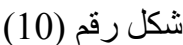

الطفل بشخصية (جون كونور)

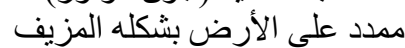
و الى الأعلى (الدوبلير ) يقوم بتمثيل الألين

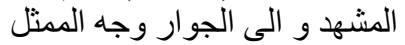

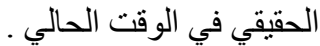

2- ت تتمتع الثخصيات الافتراضية المصنعة بالخدع السينمائية (خدع تزييف الوجوه) بمرونة في الحركة و في

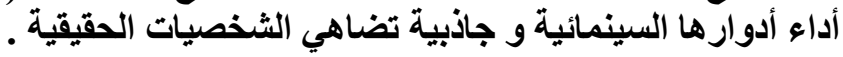

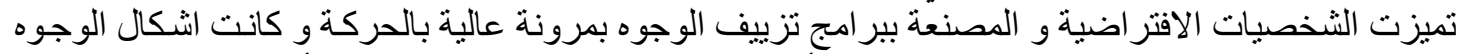

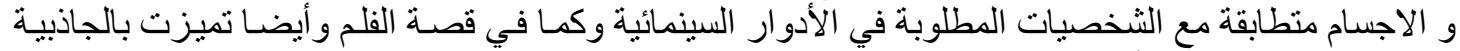

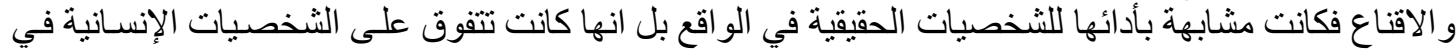
ادء المشهد من حيث الاقناع و القوة و الجمال و حسن المنظر.

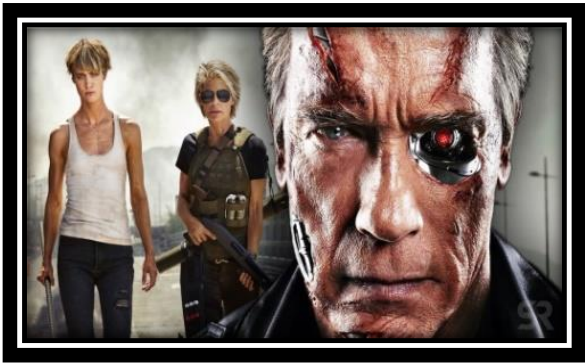

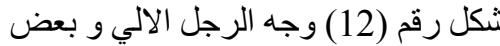
الممثلون و هم يظهرون بشكل مقارب للشخصيات الحقيقية في الفلم.

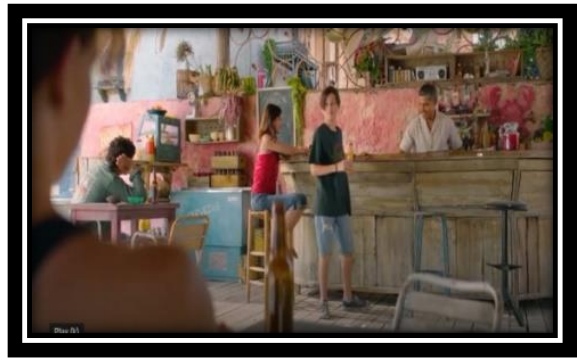

شكل رقم(11) الام و الابن و هم يتو اجدون في احد المطاعم وتبدو الشكالهم حقيقية. 


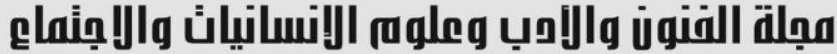

Journal of Arts, Literature, Humanities and Social Sciences www.jalhss.com

1- تنيين ان النخصيات صغيرة السن المعالجة بتقنيات الخدع السينمائية تكون اكثر مهارة و حرفية في الأداء

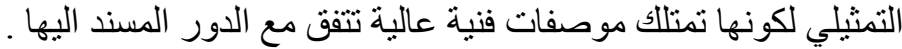

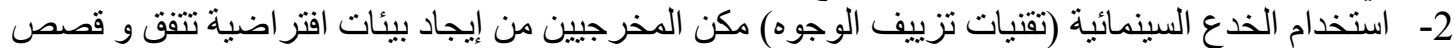

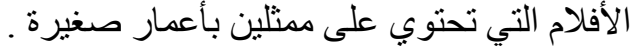

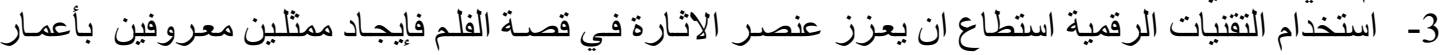
صغيرة بساهم في امتاع المشاهد و هذا صعب تحقيقه على ارض الته اقع بدون استخدام الخدع السينمائية .

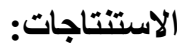

1- نجأح عمل الثخصيات الافتر اضية المعدلة ببر امج خدع التزييف دفع المخرجون الى إعطاء أدوار رئيسية

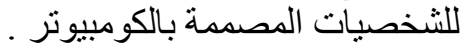

2- استخدام التقنيات الرقمية ساعد في إيجاد حلول جذريسة لمشـاهد كان من الصـب تحقيقها مثنل إعـادة ممثلين غادرو الحياة او اصبحوا كبار السن .

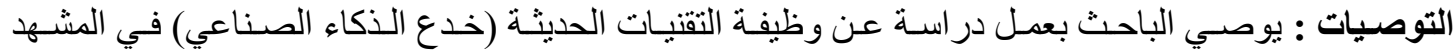
السينمائي وكيف يتم الاستفادة منها في موقع التصوير ـ درن

المقترحات: يقتر ح الباحث إضافة درس عملي ضمن منهج معاهد و كليات السينماو التلفزيون بطبق من خلاله

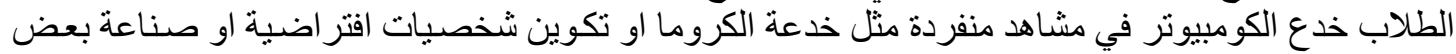

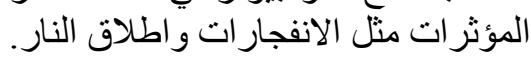

1- بهاء الدين ،طارق.(2009 ). التصوير الرقمي الحقائق و الأساسيات (ط1 )الامار ات العربية المتحدة، دار المصادر

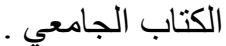

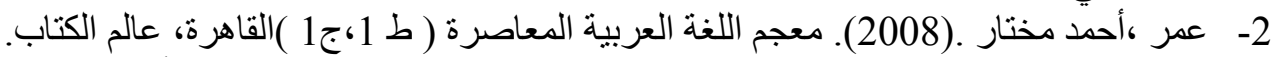

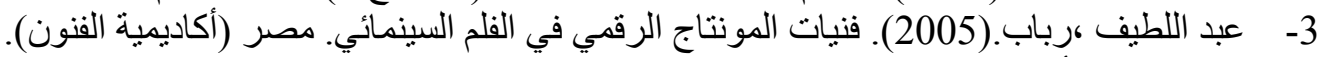

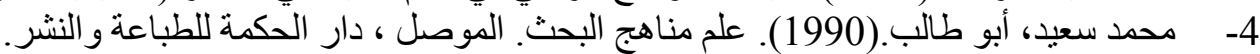

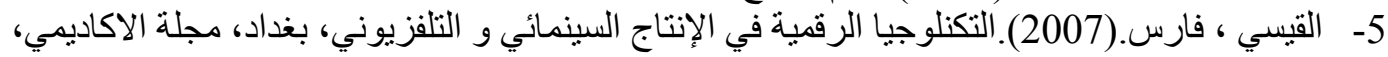

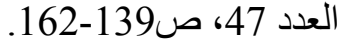

1- 1- Awaybio,S.(2020).the emerging threats of deep fake Attacks and countermeasures, Bournemouth University, UK, p2.

2- Burns, M.(2019).the De-Aging process-turning back timewitn VFX ,IBC 365, 8 November, https://www.ibc.org/trends/vfx-de-aging-tech/5188.article.

3- Davis,R.Wigging,C.(2020).Tech Factsheets for policymakers -Deep Fakes, United states of America,Harvard Kennedy school,p3.

4- Mohammed ,N.(2012). Visual Effects Cinematography is a cinematography technique from the traditional era to the digital age, Turkish journal of desing art communication tojdac, april, volume 2,issue 2,P.122-115.

5- Westerlund, M.(2019).the emergence of deep fake technology, November, vol 9,lssue 11,p 40. 